\title{
Neutral perfect fluids and charged thin shells with electromagnetic mass in general relativity
}

\author{
Victor Varela ${ }^{1}$ \\ Department of Mathematical Sciences, \\ University of Aberdeen, King's College, \\ Aberdeen AB24 3UE, Scotland, UK. \\ E-mail: v.varela@maths.abdn.ac.uk
}

\begin{abstract}
We build extended sources for the Reissner-Nordström metric. Our models describe a neutral perfect fluid core bounded by a charged thin shell, and feature everywhere positive rest mass density and everywhere non-negative active gravitational mass, as well as classical electron radius and electromagnetic total mass. We contrast our results with previously discussed models featuring similar properties at the expense of including anisotropic pressures within the fluid. Our charged thin shells are restricted by the $2 \mathrm{D}$ texture equation of state which causes the continuity of the active gravitational mass, in spite of the singularity of the energy-momentum tensor. We mention possible extensions of this study suggested by modified active mass formulae proposed in the literature.
\end{abstract}

The construction of extended sources for the Reissner-Nordström (RN) geometry has interested many researchers in Einstein-Maxwell (EM) theory. (See, for example, the partial list of contributions reviewed by Ivanov [1].) Certainly the mathematical and physical appeal of this problem is associated with the unique coupling of gravity to all types of matter and fields. However, researchers often face difficulties when they use EM equations to model the inner structure of charged particles.

Bonnor and Cooperstock [2] used a charged fluid sphere to model the electron, with a radius smaller than $10^{-16} \mathrm{~cm}$. In this study the field equations implied the existence of some negative rest mass within the fluid (a recent overview of this result is presented in [3]). Moreover, the total active gravitational mass within the charged sphere was negative, which pointed towards compatibility problems with the energy conditions and the singularity theorems of general relativity.

Ponce de León [4] presented a detailed analysis of limiting configurations allowed by the energy conditions. More recently the same author has constructed a new type of extended source for the RN geometry, with classical electron radius [5]. His relativistic version of the old Abraham-Lorentz-Poincaré model includes a charged fluid sphere with anisotropic pressures, a "pure field condition", and specific continuity conditions

\footnotetext{
${ }^{1}$ Permanent Address: Centro de Física Teórica y Computacional, Escuela de Física, Facultad de Ciencias, Universidad Central de Venezuela, Caracas, Venezuela. E-mail: vvarela@fisica.ciens.ucv.ve
} 
at the surface. The appealing properties of this electromagnetic mass model are that its active gravitational mass is non-negative everywhere and its rest mass density is positive everywhere. According to Ponce de León, pressure anisotropy must be considered whenever we wish to construct electromagnetic mass sources with such properties.

The aim of this paper is to show that extended RN sources with everywhere positive mass density, classical electron radius, electromagnetic mass, and everywhere nonnegative gravitational mass can be constructed using perfect fluid spheres with the charge concentrated at the surface. In our view, the use of unequal principal pressures in [5] has been motivated by particular boundary conditions, and cannot be considered as a necessary condition to obtain the sought properties.

We shall assume a neutral perfect fluid sphere with radius $a$, matter density $\rho$ and pressure $p$ bounded by a charged thin shell with charge $q$, tangential stress $S$, and surface matter density $K$. We adopt the Cohen and Cohen treatment of the EM equations with singular energy-momentum tensor [6], which is suitable for the study of inner and outer spherically symmetric, static geometries described in curvature coordinates.

Following [6] we use geometrical units $(c=G=1)$ and adopt a static, spherically symmetric line element written in the form

$$
d s^{2}=-A^{2} d t^{2}+B^{2} d r^{2}+r^{2}\left(d \theta^{2}+\sin \theta^{2} d \phi^{2}\right),
$$

where $A=A(r)$ and $B=B(r)$. We shall assume that $A=\sqrt{A^{2}}$ and $B=\sqrt{B^{2}}$ are everywhere positive.

We use the orthonormal base

$$
\hat{e}_{0}=\frac{1}{A} \frac{\partial}{\partial t}, \quad \hat{e}_{1}=\frac{1}{B} \frac{\partial}{\partial r}, \quad \hat{e}_{2}=\frac{1}{r} \frac{\partial}{\partial \theta}, \quad \hat{e}_{3}=\frac{1}{r \sin \theta} \frac{\partial}{\partial \phi} .
$$

The field equations in this frame take the form

$$
\begin{gathered}
8 \pi r^{2} T^{00}=\left[r\left(1-B^{-2}\right)\right]^{\prime}, \\
A^{2} B^{2}\left(1+8 \pi r^{2} T^{11}\right)=\left(r A^{2}\right)^{\prime}, \\
8 \pi A B T^{22}=\left[(r B)^{-1}(r A)^{\prime}\right]^{\prime}+\frac{A}{r^{2} B}, \\
\left(r^{2} \epsilon\right)^{\prime}=4 \pi \sigma r^{2} B,
\end{gathered}
$$

where $T^{00}, T^{11}$, and $T^{22}=T^{33}$ are the non-vanishing components of the energymomentum tensor, prime denotes a derivative with respect to $r, \sigma$ is the charge density, and $\epsilon$ is the radial electric field. We are dealing with a neutral perfect fluid sphere bounded by a charged thin shell, so $\epsilon$ vanishes inside the shell. Outside the shell we have ordinary vacuum $(\rho=p=\sigma=0)$, and the electric field reduces to

$$
\epsilon=\frac{q}{r^{2}}
$$


where the charge is given by

$$
q=4 \pi \int_{a_{-}}^{a_{+}} \sigma r^{2} B d r
$$

with $a_{-}$and $a_{+}$denoting the limit $r \rightarrow a$ taken from below and above respectively.

The energy-momentum tensor comprises contributions from the neutral perfect fluid, the charged thin shell and the external electromagnetic field. It is given by

$$
T^{00}=\rho+\rho_{s}+\frac{\epsilon^{2}}{8 \pi}, \quad T^{11}=p-\frac{\epsilon^{2}}{8 \pi}, \quad T^{22}=T^{33}=p+p_{s}+\frac{\epsilon^{2}}{8 \pi},
$$

where $\rho, p$ denote the neutral fluid mass density and pressure, respectively, and $\rho_{s}, p_{s}$ denote the mass density of the shell and the tangential stress necessary to support the shell, respectively. In this case $\rho$ and $p$ vanish outside the neutral fluid core, whilst $\rho_{s}$, $p_{s}$ have the forms

$$
\rho_{s}=K \delta(r-a), \quad p_{s}=S \delta(r-a),
$$

where the Dirac $\delta$ function is normalized to satisfy

$$
4 \pi \int_{a_{-}}^{a_{+}} \delta(r-a) r^{2} d r=1 .
$$

Cohen and Cohen use $\delta$ functions to model the thin shell contribution to the energymomentum tensor. Their procedure implies formal difficulties related to the use of distributions in general relativity. As discussed in [7], some of these difficulties are linked with the non-linearity of the field equations. The reader is referred to [8] for a discussion of energy-momentum valued distributions in the context of spherically symmetric geometries.

This thin shell formalism is characterized by its restriction to curvature coordinates and the need to consider non-linear operations on distributions. Nevertheless it shares with the more powerful formalisms described in [7] the reduction of the field equations to finite difference equations governing the discontinuities of various quantities across the shell [9]. Cohen and Cohen's formalism provides us with formulae for the determination of the physical parameters $K$ and $S$ as well.

Now we combine Eqs. (3)-(5) with Eq. (9) and integrate the arising equations in different spacetime regions. In the outer region we impose the appropriate weak field behavior for large $r$ to obtain the RN solution

$$
A^{2}=B^{-2}=1-\frac{2 m}{r}+\frac{q^{2}}{r^{2}}, \quad r>a,
$$

where $m$ is the total mass of the solution. We add the restriction $a>2 m$ to guarantee that $A^{2}$ and $B^{2}$ are positive for $r>a$.

From Eqs. (3)-(5) we get

$$
4 \pi r^{2} A B\left(T^{00}+T^{11}+T^{22}+T^{33}\right)=\left(\frac{r^{2} A^{\prime}}{B}\right)^{\prime} .
$$


Restricting our attention to the inner region, and substituting Eq. (9) into Eq. (13) we obtain

$$
4 \pi r^{2} A B(\rho+3 p)=\left(\frac{r^{2} A^{\prime}}{B}\right)^{\prime},
$$

which suggests a particular choice for the equation of state of the neutral perfect fluid.

The significance of the equation of state

$$
\rho+3 p=0
$$

was discussed by several authors in connection with global monopoles and textures (see [10] and references therein). In a recent paper [11], the simplifying power of this equation of state has been highlighted in the context of neutral perfect fluid, Majumdartype solutions of EM equations. In this paper we deal with neutral perfect fluids as well and assume that Eq. (15) is valid throughout the core. If $B(0)$ is finite and $A^{\prime}(0)=0$, then we integrate Eq. (14) to get

$$
A=A_{0}=\text { constant }, \quad r<a .
$$

Combining Eqs. (3), (5) and (9) with Eqs. (15) and (16), we find that $h=\frac{1}{r^{2} B^{2}}$ satisfies $h^{\prime}=-\frac{2}{r^{3}}$ and obtain

$$
B^{2}=\frac{1}{1-C r^{2}}, \quad r<a,
$$

where $C$ is an integration constant. Using the same field equations combined with Eqs. (16) and (17) we get

$$
\rho=\frac{3 C}{8 \pi}, \quad p=-\frac{C}{8 \pi}
$$

for the constant mass density and pressure of our neutral perfect fluid core.

The internal solution given by Eqs. (16) and (17) has vanishing Weyl tensor and constant Ricci scalar. When $0<C<\frac{1}{a^{2}}$ it actually describes a section of a spherical Einstein universe, and $B_{-}^{2}=\left(1-C a^{2}\right)^{-1}$ is bounded from above.

Integration of Eq. (3) across the shell yields

$$
\frac{1}{B_{-}^{2}}-\frac{1}{B_{+}^{2}}=\frac{2 K}{a}
$$

where $B_{+}^{2}=\left(1-\frac{2 m}{a}+\frac{q^{2}}{a^{2}}\right)^{-1}$. Thus $B^{2}$ is discontinuous at $r=a$ whenever $K$ is non zero, and Eq. (19) implies the identity

$$
m=\frac{C a^{3}}{2}+K+\frac{q^{2}}{2 a}
$$

where the provisionally independent contributions of the fluid core, the shell, and the electromagnetic field to the total mass are specified. 
Since both $B_{-}^{2}$ and $B_{+}^{2}$ are bounded from above, Eq. (4) implies the continuity of $A^{2}$ at $r=a$. Thus we combine Eqs. (12) and (16) to get

$$
A_{0}^{2}=1-\frac{2 m}{a}+\frac{q^{2}}{a^{2}}
$$

Integration of Eq. (5) across the shell yields

$$
8 \pi S \int_{a_{-}}^{a_{+}} B \delta(r-a) d r=\frac{1}{B_{+}}\left(\frac{1}{a}+\frac{A_{+}^{\prime}}{A_{0}}\right)-\frac{1}{B_{-}}\left(\frac{1}{a}+\frac{A_{-}^{\prime}}{A_{0}}\right) .
$$

Cohen and Cohen pointed out that although the integral on the left of Eq. (22) is not well defined, it can be indirectly determined by integrating Eq. (3) across the shell. This procedure yields the exact result

$$
\int_{a_{-}}^{a_{+}} B \delta(r-a) d r=\frac{1}{4 \pi a K}\left(\frac{1}{B_{-}}-\frac{1}{B_{+}}\right),
$$

which, combined with Eq. (22), provides us with a formula for the elastic stress $S$. The straightforward manipulation of an ill defined distributional operation -via the field equations- is indeed a striking feature of Cohen and Cohen's thin shell formalism.

Zloschchastiev [12] has determined equilibrium conditions for spherical charged thin shells enclosing ordinary vacuum. Using Israel's standard formalism [13], he considers shells with surface mass densities and pressures satisfying linear equations of state. Motivated by Zloschchastiev's work on $2 \mathrm{D}$ textures $[12,14]$, we impose the equation

$$
K+2 S=0
$$

and study its implications for a charged shell surrounding a neutral perfect fluid core. (We point out that Cohen and Cohen's paper [6] does not make any reference to possible equations of state satisfied by the shell parameters.)

Combining Eqs. (22) and (23) we derive a clumsy equation for $S$ as a function of $a, q, C, K$ that can be simultaneously solved with Eq. (24). This procedure provides us with an expression for the surface matter density, namely,

$$
K=-\frac{1}{2} C a^{3}+\frac{q^{2}}{2 a} .
$$

Substitution into Eq. (20) yields

$$
m=\frac{q^{2}}{a} .
$$

Thus our charged sources have classical electron radius. Notably Eq. (26) is assumed from the outset in the very different approaches developed in [5] and [12].

Using Eqs. (18) and (25) we see that the restriction $0<C<\frac{q^{2}}{a^{4}}$ guarantees the existence of a family of solutions with $\rho>0$ and $K>0$. Additionally, the charged shell that encloses ordinary vacuum (case $C=0$ ) is characterized by $K=\frac{q^{2}}{2 a}>0$.

If $a^{2}>q^{2}$ then $0<C<\frac{q^{2}}{a^{4}} \Rightarrow 0<C<\frac{1}{a^{2}}$ and we are dealing with useful solutions, i.e. the ones with $\rho>0, K>0$, and bounded $B_{-}$. We point out that the 
restrictions $a>2 m$ and $a^{2}>q^{2}$ are compatible with the approximate model of the electron discussed in [2]. (However, it should be noted that the corresponding classical radius determined with Eq. (26) is about 2817 times larger than the experimental upper limit of $10^{-16} \mathrm{~cm}$.)

Ponce de León [5] uses the Tolman-Whittaker formula

$$
M(r)=4 \pi \int_{0}^{r}\left(T^{00}+T^{11}+T^{22}+T^{33}\right) A B r^{2} d r
$$

to determine the active gravitational mass inside a sphere of radius $r$. Grøn [15] justified the use of the surface gravity $\kappa$, given by

$$
\kappa=-\frac{M(r)}{r},
$$

as a measure of (coordinate) radial acceleration for geodesic motion at instantaneous rest. The later author derived Eqs. (27) and (28) from the geodesics and Einstein equations, and discussed them when a singular shell is present. In this case a difference between the values of $M(r)$ at both sides of the shell is expected, and the integration is performed through the shell. Grøn also pointed out that negative values for $M(r)$ cause repulsive gravitation effects that may play a role in connection with elementary particle models. (See [16] for another recent consideration of Eq. (27) in connection with RN spacetimes.)

We combine the Tolman-Whittaker formula with the Cohen and Cohen method in order to determine the active gravitational mass of our solutions, including the contribution of the charged thin shell. In fact, taking into account the useful solutions discussed above and Eq. (27), we obtain

$$
M(r)=0, \quad r<a,
$$

and

$$
M(r)=q^{2}\left(\frac{1}{a}-\frac{1}{r}\right), \quad r>a .
$$

We observe that neither the neutral perfect fluid core nor the charged thin shell have active gravitational mass as a consequence of equations of state (15) and (24). Thus $M(r)$ is continuous at $r=a$, and the Maxwell field contributes with positive active gravitational mass for $r>a$. Additionally, $M(r)$ has the limit $M(\infty)=m$.

The extended sources for the RN metric discussed here include a neutral perfect fluid core bounded by a charged thin shell. The mass density $\rho$ and the surface charge density $K$ are strictly positive, and the gravitational mass $M(r)$ is everywhere nonnegative. The discontinuity imposed by the thin shell prevents the consideration of anisotropic pressures within the fluid core. Repulsive gravitational forces are totally irrelevant to these solutions.

In the limit $q \rightarrow 0$ the physical parameters $\rho, p, K$ and $S$ as well as $m$ vanish. Thus, the total gravitational mass of our solutions is built from electromagnetism only. Other electromagnetic mass sources discussed in the literature $[17,18]$ contain negative active mass and exert repulsive gravitational forces on test particles [15]. 
Using Eq. (21) in [5], we see that the combination of Eq. (15) with the conformal flatness of the internal metric determines the vanishing of the active gravitational mass for $r<a$. This interior geometry makes (coordinate) acceleration as well as tidal forces for radial geodesic motion vanish [10]. On the other hand, the $2 \mathrm{D}$ charged texture equation of state (24) suffices for the vanishing of the thin shell gravitational mass and the consequent continuity of $M(r)$ at $r=a$. (Whether the automatic vanishing of the $3 \mathrm{D}$ Weyl tensor plays a role in the vanishing of the active gravitational mass of the shell with equation of state (24) is a point that deserves further attention.) Considering that the total energy-momentum tensor is singular at $r=a$, the continuity of $M(r)$ is an intriguing feature of these models.

The above discussion is based on Eq. (27). Reservations about the use of this formula have been expressed in the past, due to its lack of invariance under a change of scale of the time coordinate [19]. On the other hand, this formula may lead to wrong results when discontinuity surfaces are included in the solutions. These observations motivated Devitt and Florides [20] to develop modified mass formulae that correct the time invariance problem and deal with static, spherically symmetric thin shells in accordance with Israel's standard formalism. A study of the useful solutions based on the Tolman-Whittaker-Devitt-Florides formalism should clarify the nature of these extended sources for the RN metric.

\section{Acknowledgements}

The author is grateful to the University of Aberdeen for hospitality while this paper was being written, and to Professor G.S. Hall for a number of useful suggestions and comments. He also thanks Mr. M. Chung for computational assistance. This work was partially supported by grants from the Universidad Central de Venezuela (CDCH and Vicerrectorado Académico), the Royal Society of Edinburgh, and the Edinburgh Mathematical Society.

\section{References}

[1] Ivanov, B.V. (2002). Phys. Rev. D 65, 104001.

[2] Bonnor, W.B. and Cooperstock, F.I. (1989). Phys. Lett. A 139, 442.

[3] Ray, S. and Bhadra, S. (2004). Int. J. Mod. Phys. D 13, 555.

[4] Ponce de León, J. (1993). Gen. Rel. Grav. 25, 1123.

[5] Ponce de León, J. (2004). Gen. Rel. Grav. 36, 1453.

[6] Cohen, J.M. and Cohen, M.D. (1969). Nuovo Cimento B 60, 241.

[7] Mansouri, R. and Khorrami, M. (1996). J. Math. Phys. 37, 5672.

[8] Pantoja, N.R. and Rago, H. E-print gr-qc/9710072.

[9] Marolf, D., Yaida, S. (2005). Phys. Rev. D 72, 044016.

[10] Dadhich, N. (1998). Gen. Rel. Grav. 30, 1133. 
[11] Varela, V. (2005). Gen. Rel. Grav. 37, 1769.

[12] Zloschchastiev, K.G. (1999). Int. J. Mod. Phys. D 8, 165.

[13] Israel, W. (1966). Nuovo Cimento B 44, 1 (erratum 48, 463).

[14] Zloschchastiev, K.G. (1999). Gen. Rel. Grav. 31, 1821. See also e-print grqc/0001002.

[15] Grøn, Ø. (1985). Phys. Rev. D 31, 2129.

[16] Barbachoux, C., Gariel, J., Marcilhacy, G. and Santos, N.O. (2002). Int. J. Mod. Phys. D 11, 1255.

[17] Tiwari, R.N., Rao, J.R., and Kanakamedala, R.R. (2000). Phys. Rev. D 30, 489.

[18] Gautreau, R. (1985). Phys. Rev. D 31, 1860.

[19] Bonnor, W.B. (1992). Class. Quantum Grav. 9, 269.

[20] Devitt, J. and Florides, P.S. (1989). Gen. Rel. Grav. 21, 585. 